\title{
Being Happy, Healthy and Whole Watching Movies That Affect Our Emotions
}

\author{
Teresa Chambel $^{1}$, Eva Oliveira ${ }^{2,1}$, and Pedro Martins ${ }^{1}$ \\ ${ }^{1}$ LaSIGE, University of Lisbon, 1749-016 Lisbon, Portugal \\ tc@il.fc.ul.pt, pedrofjfmartins@gmail.com \\ ${ }^{2}$ DIGARC, Polytechnic Institute of Cávado and Ave 4750-810 Barcelos, Portugal \\ eoliveira@ipca.pt
}

\begin{abstract}
This paper discusses the power of emotions in our health, happiness and wholeness, and the emotional impact of movies. It presents iFelt, an interactive video application to classify, access, explore and visualize movies based on their emotional properties and impact.
\end{abstract}

Keywords: Happiness, Health, Wholeness, Emotions, Movies, Emotional Impact, Technology, Entertainment, Design, HCI, iFelt.

\section{Introduction}

Emotions permeate people's daily lives, influencing the way we think and act, our health, our happiness and our sense of well-being. Recent studies demonstrated that emotions constitute a central part in the cognitive and decision making processes. That positive emotions enhance cognitive capacities and creative problem solving tasks, as well as physical health; while negative emotions, which narrow the individual's repertoire of thought and action, have a valuable survival strategy. Emotions, which are globally distributed throughout body's nervous system and cells, also have the power to influence our immune system and DNA, and the world around us. Positive Psychology is a recent research field that aims at achieving a scientific understanding of happiness, positive emotions and longevity, and has its roots in the conviction that psychology should not only help disordered people but should instead promote the quality of life of ordinary people. They aim to help people lead not only pleasant, but engaging and meaningful lives. In a sense, they share their aim with that saying: "Happy, healthy and whole in body, mind and soul", an integrated perspective of well-being, where emotions seem to play a central role. One of the greatest strengths of video is its power to generate attitudes and emotions as no other medium can. And it is becoming more and more pervasive in our lives, making it pertinent to explore its valences in inducing and supporting our empowering emotions.

This paper addresses the power of emotions, in section 2. Section 3 discusses the emotional impact of watching videos and movies. Section 4 addresses related work. The iFelt system is presented in section 5, followed, in section 6, by a user study conducted to learn about the emotional impact of movies and the usability of iFelt. The paper ends in section 7, with conclusions and perspectives for future work. 


\section{The Power of Emotions}

This section addresses the power of emotions in mental states, cognition, survival, health, happiness and wholeness. Although they are all somehow related, while contributing to our sense of well-being, we will present them in a structured way.

The Role of Positive and Negative Emotions: Many authors state that emotions are survival artifacts. They regulate biologic patterns to deal with specific environments, like hunger or fear [13], classifying things as being good or bad, or sometimes neutral [6]. However, positive emotions are not always beneficial and negative emotions are not always detrimental [11]. In evolutionary terms, it has been suggested that positive emotions are associated with opportunities and a strategy of approach, facilitating the use of internalized strategies; while negative emotions, which narrow the individual's repertoire of thought and action, have a valuable survival strategy. There is also extensive literature suggesting that it is as important to recognize our negative feelings as it is to recognize our positive feelings. For e.g., frustration or mild depression can signal that it is time to pursue a different goal.

in Health: It has been known for a long time that negative emotions are related to a higher prevalence and severity of disease, but how strong is the evidence for a link between positive emotions and health? There are a number of pathways (biological, cognitive, social...) through which positive emotions have direct and usually beneficial effects on physiological, hormonal and immune function which in turn influence health [11]. Davidson et. al., referenced in [11] demonstrated this effect obtained through meditation. Philippot et. al. [22] suggest interventions including the redirection of attention. In the early 1900's, Ed. Bach had already found and developed an approach to healing based on emotions. More recently, the new science of Epigenetics revealed that there are reserves of natural happiness within our DNA that can be controlled by us, by our emotions, beliefs and behavioral choices [3]. Also, people high in happiness or subjective well-being tend to have healthier lifestyles, more self-enhancing, productive, and more positive interpersonal experiences [11].

in Happiness and Wholeness. The Positive Psychology movement, launched in 1998 by Seligman [28], [29], [11], aims at achieving a scientific understanding of happiness, positive emotions and longevity, and effective interventions to make normal life more fulfilling for ordinary people, and not just for those with disorder or dysfunction, where the focus tended to be in psychology. It extends and has brought empirical support to the theories of the Humanistic approach, with overlaps with Existential psychology, and has influenced the more recent Coaching psychology. According to Seligman [29], happiness is related with three goals in life, to have: 1) a Pleasant life, feeling positive emotions; 2) a Good or Engaging life, based on being in flow, when time 'stops' while one engages in absorbing activities related with our higher strengths; and 3) a Meaningful life, using our signature strengths in service of something larger than ourselves. The sense of living a full life increases along the three and helps leading to health and longevity. At the crossroads of science and spirituality, authors like Braden and Lipton [3] argue that our reality code is based in the language of emotion and focused belief, giving our thought and ourselves power 
to change the conditions of healing within our bodies, and into the world around us. [10] defend that emotion is the indicator of whether we are blocking or allowing this flow towards the alignment with our true self, and a sense of wholeness.

\section{Emotional Impact of Videos and Movies}

By combining diverse symbol systems, such as pictures, texts, music and narration, video is a very rich media type, often engaging the viewer cognitively and emotionally, and having a great potential in the promotion of emotional experiences. Isen et. al. [12] attested this potential, evaluating the effect of positive affect in patients, inducted by ten-minute comedy films. Bardzell et al. [5] results suggest that emotional responses are complex but still implicated with video preferences and engagement, even in short Internet videos (more details in sec.4). The study of films as an emotion induction method has reports dated since 1916 [19], analyzing mental operations of film viewers and discussing how emotions guide the motivation of perception and the control of our attention by cinematographic narratives. According to [31], appraisal in film viewing can be seen as based on a set of illusions that are difficult to resist. It is difficult to escape the illusion of witnessing events in the fictional world, which along with two other factors explain the typically high intensity of emotion in the cinema: a certain willingness of the viewers to cooperate; and the self-amplification inherent in film-produced emotion. More recently, other researchers used films to induce emotions with different goals [8]. In media studies, the most common methods for eliciting emotions are images, music, or film based. [32] tested 11 induction methods and concluded that films were the best method to elicit emotions, positive and negative.

\section{Related Work}

We present a review of work that more closely relates with our iFelt system.

Models and Representations of Emotions. There is a diversity of perspectives on the emotional properties of video, regarding its content or the emotional impact it has on viewers. From the directors' point of view, there are a number of cinematographic techniques [2] to induce the emotional environment, like shots duration, lightning conditions, color and movements. From the viewer and content point of view, there are two main models of emotion: 1) The Dimensional Model [26] is based on a two dimensional spatial circumplex: arousal (intensity) and valence (polarity); 2) The Categorical Model defines emotions as discrete states that identify a certain behavior and experience. Ekman [7] identified six basic emotions based on facial expressions recognized across cultures: anger, disgust, fear, joy, sadness and surprise. There is a correspondence of these emotions in Russel's circumplex. The Appraisal Model is also categorical, defined as the evaluation of the interaction between someone and their goals, beliefs and the environment [27]. Plutchick [24] used both categorical and dimensional models and defined a 3D model (polarity, similarity, intensity) with eight primary emotions: anger, fear, sadness, disgust, surprise, anticipation, trust, and joy, represented around the center, in colors, with the intensity as the vertical dimension. 
Emotional Classification of Movies and their Impact. Video content analyzes and classification deals with techniques to extract info from video, or users, to find meaningful segments. Some techniques use automatic processing, others are manual. The extraction of content-based emotional info expressed in videos has been based on low-level features like color, texture, lightning, motions, sounds, rhythm, lexicon, etc. inspired by cinema theorists tools [9], resulting from directors intention for felt emotions. The classification of movies by its actual affective impact has recently been the focus of some studies, using: biometric methods based on physiological signals, such as respiration, heart rate, blood pressure, electromyograms, and galvanic skin response; or recognition of facial emotional expressions [17]. Works have been recently developed to prove that films can be emotional inductors, helping psychologists in specific treatments [14], or to automatically index, search [30], or summarize [18] videos, according to the emotional impact, that tend to be significantly different among the viewers. [5] studied user emotions and engagement with internet videos, using physiological measures to assess valence and arousal, emotional self-reports, and prose reviews, concluding that emotion is implicated in video preferences.

Accessing and Visualizing Movies and Videos. IMDB (.com) provides info about actors, directors, genres, film ratings, etc. Others, like Netflix, also allow accessing and watching movies. YouTube is probably the most famous website to publish and watch videos, search, comment, share, and get recommendations. But none of these systems support emotional info, and they do not explore the visualization of video spaces much further then through lists. Film Finder [1] supported users to search and view films based on duration, genres, titles, actors and directors, using starfield graphics based on date and popularity. However, most visualization tools and apps do not address video. Among the exceptions, the most related to our work are: 1) the YouTube 2D view representing videos as circular scattered still images, allowing for visual neighborhood navigation based on similarity; and 2) Video Sphere (bestiario. org/research/videosphere), representing TED's videos around a 3D sphere connected by semantic links. In our previous works: we provided interactive 3D visualization and navigation of videos [25], to explore cultural and aesthetic properties of videos and videos spaces; and a 2D interactive system based on a physical particles system [16] to visualize and explore videos based on color dominance, rhythm and movement. These systems address video visualization but not emotions.

Eliciting and Visualizing Emotions. In this context, there are some recent works, but not so much on video. We Feel Fine (.org) harvests human feelings from weblogs, searching feelings after "I feel" and "I am feeling" and info about the author and local weather when it was written. The interface is based on a colored physical particles system, representing expressed feelings. Mappiness (.org.uk) maps happiness across space in UK, to better understand how people's feelings are affected by their current environment, including air pollution, noise, and green spaces, by prompting users a couple of times a day, on their iPhones. Synesketch (.krcadinac.com) is a textual emotion recognition and visualization software based on synesthesia, dynamically transferring text into animated visual patterns, using colored squares, and moving particles. The Emotionally\} Vague (.com) project addresses the relation of body and emotion and how people feel emotions (e.g. body location and associated colors). 


\section{The iFelt System}

iFelt is an interactive web video application that allows to catalog, access, explore and visualize emotional information about movies. It is being designed to explore the affective dimensions of movies in terms of their properties and in accordance with users' emotional profiles, choices and states, in two main components:

1. Emotional Movie Content Classification aims to provide video classification and indexing based on emotions, either expressed in the movies (objective emotions), or felt by the users (subjective emotions), as dominant emotions at the level of the movie or the movie scenes. Objective emotions are being classified with the aid of video low-level feature analysis, combined with audio and subtitles processing in our VIRUS research project [15]; while subjective emotions can be classified manually, or automatically recognized with biometric methods based on physiological signals, such as respiration, heart rate and galvanic skin response, employing digital signal processing and pattern recognition algorithms, inspired by statistical techniques used by [23]. This process and its results are thoroughly described in [21].

In the IFelt system, we started by using the categorical labels: happy, sadness, surprise, fear, anger and disgust, for the classification of both content and users' emotions, mainly due to the facts that: 1) the differentiations of a larger range of emotions by physiologic patterns is still limited; and 2) the Ekman's basic emotions are well known. In iFelt, emotions are detected or recognized along time, allowing to identify objective and subjective emotional scenes, along with the dominant emotion expressed or felt in each movie. Although work is already being done from both perspectives, in this first prototype, the focus was more directed towards the subjective perspective, where we consider three different views: 1) My view: represents videos classified by the emotions felt by the current user while watching each movie; 2) All Users view: computes the dominant emotions among those felt by all the viewers of each video; 3 ) The Directors view: represents the emotions that the movie's director, or possibly film expert, expects users to feel while watching it.

2. Emotional Movie Access and Exploration. This component aims to provide video access and visualization based on their emotional properties and users' emotions and profiles. The first prototype is focused on the subjective emotions perspective, to explore and evaluate the emotional paradigm, on top of which we will later add the other perspectives. The design options are thoroughly addressed in [20]. Here we shortly mention that our representation is based on colors, inspired in the model of Plutchik [24], representing anger, disgust, fear, sadness, surprise and happiness, by the colors pink, purple, green, blue, light blue, and yellow, and that we consistently adopt round shapes, and circular organizations inspired by the 2D Russel's circumplex [26]. Next, we will briefly highlight the main features of iFelt.

In the Movie Space, the user gets a view over the movies existing in iFelt, with info about their dominant emotions, in addition to the traditional info of title, etc. This can be the view of all the videos or a selection resulting from a previous search. From here, the user may navigate to any of the represented movies to watch it (Fig.1 a-b). In the current prototype, users can choose from: the 1) Emotional Wheel, where movies are represented by a colored circle, with their dominant emotion color, placed on the 
wheel accordingly in six emotional regions, and having the distance to the center representing the level of emotion dominance. Different versions were designed and tested, to increase the perceptiveness and effectiveness of this representation [20]; and the 2) Movie Title List, with an image and the title of the movie preceded by a colored circle representing the movie's dominant emotion (e.g. user profile Fig.1d).

In the Movies Emotional Scenes Space, users can view the scenes of the movies based on dominant emotions, by colored circles, with size reflecting dominance of emotion, and grouped by dominant colors. When the cursor is over one circle, the circles of the same movie in the different emotions are highlighted while the others dim (Fig.1c). When clicked, the user is directed to that individual movie, similar to Fig.1b), but presenting only the scenes with the selected emotion, as an emotional summary, e.g. scenes where most users felt sad. Both movie space and scenes space can be viewed from the three views: My, All Users (default) and Director's view.

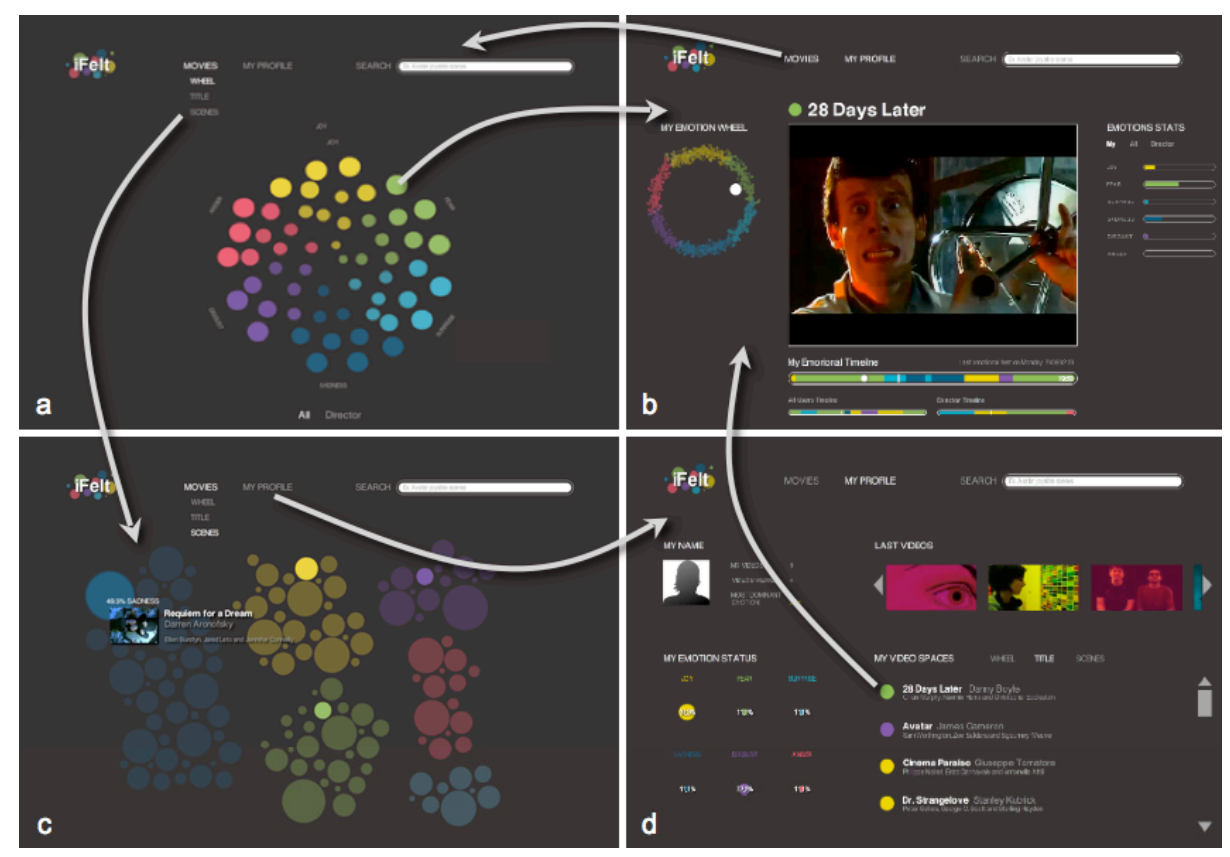

Fig. 1. iFelt: a) Movies Space (in movie wheel); b) Movie Profile; c) Emotional Scenes Space; d) User Profile (in TitleList)

At the Individual Movie Level, or Movie Profile, the movie can be watched, and there is info about its dominant emotions and emotional scenes, through: 1) The Most Dominant Emotion, as a big colored circle on top of the video; 2) The Dominant Emotions, in the current view, represented by the percentage of dominance of each emotion in the movie (bars to the right of the video, in Fig.1b); 3) The Emotional Timelines: the emotional scenes along time, below the video. The top timeline represents the emotions in the current view (mine, all users, or director's), while the bottom ones represents the other two, to help gain awareness. We explored their design to 
ease having one view as the dominant at each moment and to ease the comparison, when required [20]. These timelines also allow to access scenes based on their dominant emotions: by clicking the timeline in the chosen color; 4) The Current Emotion is represented: a) as a pointer traveling along the Timeline; and b) in a Circle of Emotions, as an animated white big dot moving to the current emotion.

From the User Emotional profile, the current users in iFelt can get info about movies classified from their own perspective or view: 1) My Personal Info: photo and name, most dominant emotion felt in the movies already classified from My perspective, and the date of the last classification (Fig.1d, top left); 2) My Dominant Felt Emotions: represented by colored circles, with size reflecting the \% of felt emotion dominance in the movies classified by me (Fig.1d, bottom left); 3) My Last Classified Movies: in the representation in Fig.1d, top right, each movie is represented by an image of the movie, tainted with a color filter corresponding to the dominant emotion felt by me; 4) My Classified Movies Space: similar to the Movies Space, but presenting only my movies, classified through my felt emotions - in title list view in Fig.1d, bottom right; 5) My Classified Emotional Scenes: similar to the Emotional Scenes, but presenting my scenes (alternate with Movie Space, when selected).

\section{User Study}

This section presents the objectives, method and results of the user study conducted to 1) learn about emotional impact in Movie Watching; and 2) evaluate iFelt's usability. We performed an evaluation based mainly on semi-structured interviews, and user observation while they performed pre-defined tasks. After each task, users were confronted with usability questions and the opportunity to provide comments and suggestions. Finally, users were asked to answer to questions regarding their attitudes, awareness and preferences about the emotional impact of movies. We had 10 participants aged 21-56, 6 female, and computer literate. Results are presented next.

1. Emotional Impact in Movie Watching. We asked viewers a few questions that are presented in tables 1-3, along with the results. Questions Q1-Q3, in table 1, aimed at the awareness and attitudes. Viewers strongly agreed (4.6 in a 1-5 scale: totally disagree - totally agree) that watching a movie can fill one's soul, or make one sad (Q1). They quite often feel the need to watch movies (4.2 in Q3, in a 1-5 scale: neveroften), and sometimes turn to movies to achieve a specific emotional state (3.1 in Q2).

Table 1. Awareness \& Attitudes about Emotional Impact

\begin{tabular}{|c|c|c|}
\hline agree: scale (1-5) & Std & Mean \\
\hline Q1. Do you agree that watching a movie can fill one's soul, or make one sad? & 0.52 & 4.6 \\
\hline Q2. How often: you turn to movies to achieve a specific emotional state? & 0.99 & 3.1 \\
\hline Q3. How often: you feel the need to watch movies? & 0.63 & 4.2 \\
\hline
\end{tabular}

Concerning the relation of genres and felt emotions (Q4-Q5 in table 2), we asked viewers what genres made them feel each of the emotions presented. We did not list the genres, to let them freely mention the most relevant. The most similar answers 
associated joy and happiness with comedy, sadness with drama, and scare/fear with terror movies. Next, action movies make most viewers feel energetic, what comedies does for fewer; most female feel depressed with drama, while most male feel depressed with romance; and disgust tends to come with terror movies, but also with those involving blood and documentaries $(2 \mathrm{M})$, and science (1F). Females get more inspired or motivated with romance, drama and action, while males get their inspiration and motivation from action and docs. Somehow opposite to the results on feeling depressed, romance makes some females feel joyful, happy, motivated and inspired, and 1 female and 1 male feel sad. As for the most wanted or preferred genre (Q5), females prefer drama and romance, while male prefer action, comedy and suspense.

To learn about preferred films and emotions, Q6-Q8 were asked as open questions (table 3). The most wanted emotion when selecting a movie (Q6) was surprise or suspense to half of the males and feeling good and fun to the others, while dreaming, inspiration or motivation were mentioned by half of the females, 2 mentioned related feelings: of making them think, wander and dream, and 1 mentioned happiness.

Table 2. Genres and Felt Emotions

\begin{tabular}{|c|c|c|c|c|c|c|c|c|c|}
\hline $\begin{array}{l}\text { Q4. What } \\
\text { movie genre } \\
\text { makes you feel: }\end{array}$ & :0્0ี & 芯 & 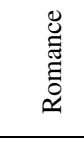 & 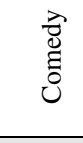 & 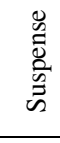 & 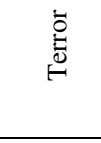 & $\stackrel{\circ}{\oplus}$ & $\dot{\circ}$ & $\begin{array}{l}\bar{\Xi} \\
\bar{\Xi}\end{array}$ \\
\hline energetic & $4 \mathrm{~F} 2 \mathrm{M}$ & & & $1 \mathrm{~F} 1 \mathrm{M}$ & $1 \mathrm{M}$ & & & & \\
\hline depressed & & $3 \mathrm{~F} 1 \mathrm{M}$ & $1 \mathrm{~F} 3 \mathrm{M}$ & & & $1 \mathrm{~F}$ & & & \\
\hline inspired & $2 \mathrm{~F} 1 \mathrm{M}$ & $3 \mathrm{~F}$ & $2 \mathrm{~F}$ & $1 \mathrm{M}$ & & & $1 \mathrm{~F}$ & $1 \mathrm{M}$ & $1 \mathrm{M}$ horses \\
\hline motivated & $1 \mathrm{~F} 2 \mathrm{M}$ & $1 \mathrm{~F}$ & $2 \mathrm{~F}$ & $1 \mathrm{~F}$ & $1 \mathrm{M}$ & & & $1 \mathrm{M}$ & 1F politics \\
\hline joyful happy & & & & $4 \mathrm{~F} 4 \mathrm{M}$ & & & & & \\
\hline & & $6 \mathrm{~F} 3 \mathrm{M}$ & $1 \mathrm{~F} 1 \mathrm{M}$ & & & & & & \\
\hline $\begin{array}{l}\text { scared } \\
\text { disgusted }\end{array}$ & & & & & $1 \mathrm{M}$ & $\begin{array}{c}6 \mathrm{~F} 3 \mathrm{M} \\
3 \mathrm{~F} 2 \mathrm{M}\end{array}$ & & $1 \mathrm{M}$ & 1F science \\
\hline $\begin{array}{l}\text { Q5. most wanted } \\
\text { genre? }\end{array}$ & $1 \mathrm{~F} 1 \mathrm{M}$ & $3 \mathrm{~F}$ & $3 \mathrm{~F}$ & $1 \mathrm{~F} 1 \mathrm{M}$ & $1 \mathrm{M}$ & & & & $1 \mathrm{~F}$ advent \\
\hline
\end{tabular}

Table 3. Preferred Movies and Emotions (Fx \& Mx: $\mathrm{x}=\mathrm{age})$

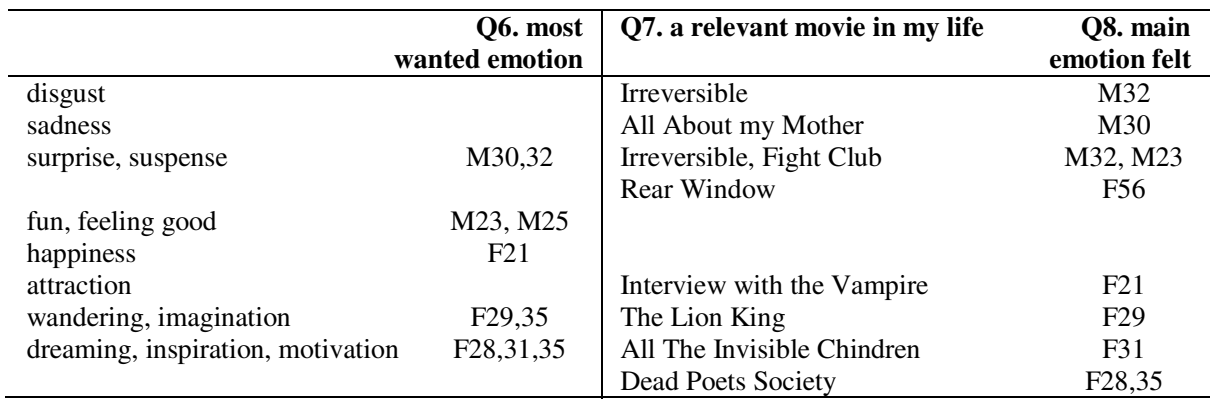


When asked about a relevant movie in their lives (Q7-Q8), the movies and the associated main felt emotion align to a reasonable extent with the preferred emotions. Also interesting to note that in the imagination and motivation movies, answers were somehow more inspired: F29 "enjoyed wondering about the story", while F35, after watching the Dead Poets Society at 13, felt she "was going to conquer the world".

2. iFelt Usability Evaluation. The usability dimensions underlying the USE questionnaire (usesurvey.com): Usefulness (of emotional info and features in iFelt), Satisfaction (fun, good experience), and Ease of use, also present in the requirements and design rationale, informed the main objectives and structure of the evaluation of iFelt. Users found Movies Space very useful (U:4.5 on average in 1-5), satisfying (S:4.6) and easy to use (E:4.7). Overall usability of the Movie Profile was U:4.7, S:4.4,E:5; Scene Space had U:4.4, S:4.2, E:4.1, and the User Emotional Profile U:4.7, $\mathrm{S}: 4.1, \mathrm{E}: 4.3$. For a more thorough discussion of the design options, the tasks performed and the results of the usability evaluation of all the iFelt features refer to [20].

\section{Conclusions and Perspectives}

On the usability evaluation, iFelt was perceived as useful, satisfactory and easy to use. Most appreciated features included the possibility to access movies and scenes based on the emotions felt, and to access and compare their own emotional views with those of other users and directors, at the level of the movies and the scenes, and along emotional timelines [20]. On the user study on the emotional impact of movies, we observed that inquired viewers strongly agreed that watching a movie can fill one's soul or make one sad, they quite often feel the need to watch movies, and sometimes turn to movies to achieve a specific emotional state. Concerning the preferences and relation of emotions and movie genres, it was interesting to note that the difference between female and male answers somehow match some traits commonly associated with each genre, for e.g. in terms of romance vs action, or dreaming vs down to earth. This separation in genders emerged from the results, and although not generalizable, it is interesting to note the tendencies, and that they align for e.g. with [4] where females reported greater preference for happy-mood films than males, whereas males had greater preference for high-arousal films. It was also interesting to note some tendency in preferences towards emotions like surprise, fun, feeling good, happiness, and mostly, imagining, dreaming, inspiration and motivation. This is somehow aligned with the levels or goals related with leading a happy life [29]: increasing positive emotions is not enough, it requires engagement and meaning.

As future directions, we are considering to add the appraisal model in the emotion classification, in a manual perspective and, whenever possible, as part of an automatic process, that also needs to be tuned. This model gives a wider range of emotions, as those that emerged as important in our user study, also to be complemented with models of engagement and enjoyment. Other features include: extending the concept of movies summarizing, searching or recommending based on users current emotional states, profiles, or defined emotional criteria; to find movies by example: with emotional timelines similar to that of a given movie; and to include support for historical emotional info gathered along time, in the different perspectives. We also intend to do 
user studies with a wider range of viewers, including psychologists, movie experts, directors and actors (some already showed interest in knowing the emotional impact of movies), to increase our awareness in ways that might inform future design options in iFelt, and to learn how this tool may contribute to add to people's lives.

Acknowledgements. This work is partially supported by FCT through LaSIGE Multiannual Funding, PROTEC (SFRH/BD/49475/2009) \& VIRUS project (PTDC/EIA-EIA/101012/2008).

\section{References}

1. Ahlberg, C., Truvé, S.: Tight coupling: Guiding user actions in a direct manipulation retrieval system. In: People and Computers: Proc. of HCI 1995, pp. 305-321 (1995)

2. Arijon, D.: Grammar of the film language. Focal Press (1976)

3. Baird, J., Nadel, L., Lipton, B.: Happiness Genes: Unlock the Positive Potential Hidden in Your DNA. New Page Books (2010)

4. Banerjee, S., Greene, K., Krcmar, M., Bagdasarov, Z., Ruginyte, D.: The role of gender and sensation seeking in film choice: Exploring mood and arousal. Journal of Media Psychology: Theories, Methods, and Applications 20(3), 97-105 (2008)

5. Bardzell, S., Bardzell, J., Pace, T.: Understanding Affective Interaction: Emotion, Engagement, and Internet Videos. In: Proc. of IEEE ACII, Amst., NL (September 10-12, 2009)

6. Damasio, A.: Descartes' Error. Harper Perennial (1995)

7. Ekman, P.: Are there basic emotions? Psychological Review 99(3), 550-553 (1992)

8. Gross, J., Levenson, R.: Emotion Elicitation Using Films. Cognition \& Emotion 1(9) (1995)

9. Hanjalic, A., Xu, L.-Q.: Affective video content representation and modeling. IEEE Transactions on Multimedia 7(1) (2005)

10. Hicks, E., Hicks, J.: The Astonishing Power of Emotions: Let Your Feelings Be Your Guide. Hay House (2007)

11. Huppert, F.: Positive emotions and cognition: developmental, neuroscience and health perspectives. In: Forgas, J.P. (ed.) Hearts and Minds: Affective Influences on Social Cognition and Behavior, Psychology Press, New York (2006)

12. Isen, A.M., Daubman, K.A., Nowicki, G.P.: Positive affect facilitates creative problem solving. Journal of Personality and Social Psychology 52, 1122-1131 (1987)

13. James, W.: What is an Emotion? Mind 9(34), 188-205 (1884)

14. Kreibig, S., Wilhelm, F., Roth, W., Gross, J.: Cardiovascular, electrodermal, and respiratory response patterns to fear- and sadness-inducing films. Psychophysiology 44(5), 787-806 (2007)

15. Langlois, T., Chambel, T., Oliveira, E., Carvalho, P., Marques, G., Falcão, A.: VIRUS: Video Information Retrieval Using Subtitles. In: Proc.of Academic MindTrek 2010 (2010)

16. Martinho, J., Chambel, T.: ColorsInMotion: Interactive Visualization and Exploration of Video Spaces. In: Proc. of Academic MindTrek 2009, Tampere, Finland (SeptemberOctober 2009)

17. Mauss, I., Robinson, M.: Measures of emotion: A review. Cognition \& Emotion 23(2), 209-237 (2009)

18. Money, A., Agius, H.: Analysing user physiological responses for affective video summarization. Displays 30(2), 59-70 (2009) 
19. Münsterberg, H.: The film: A psychological study: The silent photoplay in 1916. Dover Public, New York (1970)

20. Oliveira, E., Martins, P., Chambel, T.: iFelt: Accessing Movies Through Our Emotions. In: Proceedings of EuroiTV 2011, Lisbon, Portugal (June 29-July 1, 2011)

21. Oliveira, E., Benovoy, M., Ribeiro, N., Chambel, T.: Towards Emotional Interaction: Using Movies to Automatically Learn Users' Emotional States. In: Campos, P., Graham, N., Jorge, J., Nunes, N., Palanque, P., Winckler, M. (eds.) INTERACT 2011, Part I. LNCS, vol. 6946, pp. 152-161. Springer, Heidelberg (2011)

22. Philippot, P., Baeyens, C., Douilliez, C., Francart, B.: Cognitive regulation of emotion: Application to clinical disorders. In: Philippot, P., Feldman, R.S. (eds.) The Regulation of Emotion. Laurence Erlbaum Associates, New York (2004)

23. Picard, R., Vyzas, E., Healey, J. Toward Machine Emotional Intelligence: Analysis of Affective Physiological State. IEEE Trans. on Pattern Analysis \& Machine Intel. (2001)

24. Plutchik, R.: Emotion: A psychoevolutionary synthesis. Harper \& Row, New York (1980)

25. Rocha, T., Chambel, T.: VideoSpace: a 3D Video Experience. In: Proceedings of Artech 2008, 4th International Conference on Digital Arts, Porto, Portugal (November 2008)

26. Russell, J.: A circumflex model of affect. Journal of Personality and Social Psychology 39, 1161-1178 (1980)

27. Scherer, K.: What are emotions? and how can they be measured? Social Science Information 44(4), 695 (2005)

28. Seligman, M., Csikszentmihalyi, M.: Positive Psychology: An Introduction. American Psychologist 55(1), 5-14 (2000)

29. Seligman, M.: Martin Seligman on positive psychology, TED Talk (February 2004)

30. Soleymani, M., Chanel, C., Kierkels, J., Pun, T.: Affective Characterization of Movie Scenes Based on Content Analysis and Physiological Changes. In: Int. Symp. on MM (2008)

31. Tan, E.S.: Film-induced affect as a witness emotion. Poetics In Emotions and Cultural Products 23(1-2), 7-32 (1995)

32. Westermann, R., Spies, K., Stahl, G., Hesse, F.W.: Relative effectiveness and validity of mood induction procedures: a meta-analysis. Eur. Journal of Social Psychology 26(4), 557-580 (1996) 